\title{
Caracterização da queixa escolar no Centro de Psicologia Aplicada da Universidade Federal de Juiz de Fora
}

\author{
A characterisation of poor school performance at the \\ centre for applied Psychology of the Universidade \\ Federal de Juiz de Fora, Brazil
}

\author{
Marisa Cosenza RODRIGUES \\ Ana Paula Soares CAMPOS' \\ Isabela Andrade FERNANDES
}

\begin{abstract}
Resumo
A queixa escolar vem se constituindo, historicamente, como um dos motivos mais frequentes de encaminhamentos de crianças para os serviços-escola de Psicologia, traduzindo, pela sua complexidade, um desafio para a formação e práticas no âmbito da psicologia escolar/educacional. Diante da necessidade de aperfeiçoar o atendimento à clientela e o processo de triagem, o presente estudo objetivou analisar os prontuários referentes à queixa escolar de pacientes atendidos no Centro de Psicologia Aplicada da Universidade Federal de Juiz de Fora, Minas Gerais, Brasil, num total de 1590 documentos, no período de 1996 a 2009. Os resultados quanto à clientela evidenciaram a prevalência de meninos na faixa entre 9 e 13 anos de idade, com predomínio de encaminhamentos oriundos de escolas de periférias da cidade. Quanto à queixa, constatou-se expressiva coocorrência de problemas comportamentais e de aprendizagem. Os dados permitem uma reflexão crítica acerca da queixa escolar e de alguns fatores contextuais, bem como a necessidade de aperfeiçoamento da ficha de triagem.
\end{abstract}

Unitermos: Baixo rendimento escolar. Psicologia aplicada. Psicologia educacional.

\begin{abstract}
Poor school performance has historically constituted one of most frequent reasons for student referrals to applied psychology centers and, due to the complexity involved, has posed a challenge to academic development and practices in the field of educational and school psychology. Faced with the need to improve customer service and the screening process, this study aimed to examine a total of 1,590 school records registering poor performance for the period of 1996-2009 among patients to the Centre for Applied Psychology of the Federal University of Juiz de For a, Minas Gerais, Brazil. Results revealed a prevalence of boys aged between 9 and 13 and a predominance of referrals from schools located in the city's outskirts. Poor school performance was found to often involve behavioural problems and learning difficulties. The data allowed us to make a critical analysis of poor school performance and other contextual factors, as well as the need for an improvement in the screening process.
\end{abstract}

Uniterms: Underachievement. Educational psychology. Applied psychology.

- Universidade Federal de Juiz de Fora, Curso de Psicologia, Departamento de Psicologia. R. José Lourenço Kelmer, s/n., Campus Universitário, São Pedro, 36036-330, Juiz de Fora, MG, Brasil. Correspondência para/Correspondence to: M.C. RODRIGUES. E-mail: <rodriguesma@terra.com.br>. 
O encaminhamento de crianças e adolescentes com queixa escolar aos serviços-escola das universidades tem sido cada vez mais expressivo nas últimas décadas, redundando em reflexões críticas e questionamentos acerca das práticas de supervisão de estágio e formação acadêmica na área de psicologia escolar/educacional. Diante da necessidade de aperfeiçoar a triagem, de forma crítica e menos reativa, e melhorar a qualidade dos atendimentos a essa clientela que procura os serviços prestados pelo curso de Psiclogia, cresce o número de pesquisas que buscam investigar não só a especificidade das queixas escolares, mas também, de forma mais geral, a caracterização da clientela e a tipologia dos serviços oferecidos (Ancona-Lopez, 1983; Barbosa \& Silvares, 1994; Boarani \& Borges, 1998; Cunha \& Benetti, 2009; Graminha, 1994; Graminha \& Martins, 1994; Levandowski \& Scortegagna, 2004; Marturanno, Linhares, Loureiro \& Machado, 1997; Melo \& Perfeito, 2006; Nakamura, Lima, Tada \& Junqueira, 2008; Santos, 1990).

Na literatura encontram-se, portanto, tanto pesquisas mais amplas, que buscam caracterizar o perfil da clientela que procura os serviços públicos e privados de Psicologia, quanto pesquisas mais específicas relacionadas à queixa escolar. Para Romaro e Capitão (2003), a caracterização da clientela nas clínicas-escolas pode viabilizar o redirecionamento das modalidades de atendimento ao público que busca auxílio psicológico.

Na vertente dos estudos mais gerais, Romaro e Capitão (2003) apontam, por exemplo, que entre as crianças e os adolescentes predominam os relatos de queixas múltiplas, sendo mais frequentes as dificuldades escolares para as crianças, e as dificuldades no relacionamento interpessoal para adolescentes. Estudo semelhante realizado por Louzada (2003), que buscou delinear o perfil da clientela do Núcleo de Psicologia Aplicada da Universidade Federal do Espírito Santo (NPA/UFES), encontrou um equilíbrio quantitativo entre os sexos e maior concentração de indivíduos na faixa etária de 20 a 29 anos. A queixa genérica de "nervosismo" foi identificada como predominante nos protocolos analisados, sendo que os problemas familiares mostraram-se associados aos pacientes adultos e Dificuldade de Aprendizagem (DA), às crianças e adolescentes. Campezatto e Nunes (2007) realizaram um levantamento 242 sobre a população que buscou atendimento em oito clínicas-escolas da região metropolitana de Porto Alegre no ano de 2004. O estudo também identificou as DA entre as queixas mais frequentes, seguidas de queixas relacionadas a problemas comportamentais, dificuldades afetivo-emocionais e nos processos cognitivos, sendo a maior ocorrência de registros para o sexo feminino. É interessante destacar, nessa direção mais ampla, que estudos de revisão abarcando a década de 1960 e fins dos anos 1990 (Levandowski, 1998; Silvares, 1996), encontraram, expressivo encaminhamento de clientela infantil (predominantemente escolares, do sexo masculino, entre seis e dez anos de idade) com queixas principais de problemas de aprendizagem ou relacionadas ao contexto escolar. O trabalho realizado por Savalhia e Nunes (2007), que revisaram pesquisas realizadas entre 1998 e 2006, indicou o mesmo quadro quanto ao perfil de encaminhamento de criança, expressando, porém, a especificidade de problemas cognitivos dentre os motivos de encaminhamento.

Embora os estudos envolvendo uma caracterização mais geral forneçam um panorama sociodemográfico da clientela e subsídios relevantes para a rotina dos serviços de psicologia aplicada, o conhecimento e a análise das queixas escolares contribuem para melhorar o suporte referente aos atendimentos específicos dessa clientela. De forma mais geral, pode-se considerar queixas escolares "como aquelas referentes às dificuldades no âmbito do processo de escolarização" (Souza, 1997, p.24). Para Braga e Morais (2007), as queixas escolares envolvem encaminhamentos de crianças e adolescentes que incluem questões relacionadas à escola, seja de ordem pedagógica ou comportamental.

Na perspectiva de trabalhos focalizando a queixa escolar, Levandowski e Scortegagna (2004) evidenciaram que $36 \%$ das queixas apresentadas referem-se aos problemas de aprendizagem, 31\% aos problemas de comportamento, 29\% aos problemas emocionais e 15\% a outras questões escolares, corroborando o estudo de Romaro e Capitão (2003), que indicou predomínio de queixas múltiplas nos encaminhamentos.

Estudos recentes têm identificado diferenças entre as queixas de meninos e meninas. $O$ estudo de meta-análise realizado por Boaz e Nunes (2009), que objetivou contextualizar as queixas infantis enca- 
minhadas às clínicas-escolas brasileiras nas últimas três décadas indicou, quanto ao sexo que a maior demanda de atendimento psicológico foi de meninos, na faixa etária de 6 a 10 anos, sendo as dificuldades escolares e problemas comportamentais as queixas mais frequentes nessa clientela. Barbosa e Silvares (1994) encontraram também predominância do sexo masculino, entre 6 e 9 anos, com queixas de distúrbios de comportamento ou dificuldades escolares. Cunha e Benetti (2009) evidenciaram que a maior demanda por atendimento psicológico foi também de meninos nessa mesma faixa etária de 6 a 9 anos, com predomínio de queixas múltiplas de problemas afetivos e de comportamento. Queixas múltiplas também apareceram como categoria de maior incidência no trabalho de Nakamura et al. (2008), que também evidenciou procura mais expressiva por parte de escolares do sexo masculino, com idade entre 5 e 14 anos. Os autores identificaram a necessidade de uma orientação mais adequada aos estagiários de psicologia escolar que atendem no serviço-escola de Psicologia da Universidade Federal de Rondônia (UNIR). Cabral e Sawaya (2001) também constataram em pesquisa realizada na região Sudeste que, no caso de crianças e adolescentes encaminhados para atendimento psicológico, 69\% apresentavam queixas escolares. Merg (2008), numa revisão dos estudos sobre a clientela infantil das clínicas-escola de Psicologia dos últimos trinta anos, sintetiza que não houve modificações significativas ao longo do tempo em relação á esse perfil de encaminhamentos. Ressalta-se, todavia, que a demanda de queixas escolares não têm sido exclusividade dos serviços universitários de Psicologia. Como observa Muñiz (2001), a procura por atendimentos também vem crescendo nos serviços públicos de saúde. No estudo realizado pelo autor, as DA e adaptação escolar compuseram 35\% das motivações para consultas pediátricas e 45\% dos atendimentos mais gerais em saúde mental.

Souza (2007) argumenta que a questão da queixa escolar vem sendo discutida desde os anos de 1980, identificando-se os estudos de Moysés e Collares (1992) e de Collares e Moysés (1996) como marcos referenciais, pois já alertavam para o foco restritivo da queixa sobre o aluno, bem como para os riscos de medicalização/ patologização dos problemas vinculados ao processo de escolarização. Atualmente, como salientam Nakamura et al. (2008), embora ainda sejam hegemônicas as práticas de atendimento clínico da queixa escolar, observa-se a emergência de uma perspectiva mais contextualizada, que busca a integração dos diversos fatores envolvidos nessa queixa, haja vista sua multicausalidade e complexidade.

Há um consenso entre os autores de que o levantamento e a análise da queixa escolar possibilitam discussões acerca da prática do psicólogo escolar/ educacional, do papel do professor e da escola como espaço institucional corresponsável, das concepções críticas em relação às dificuldades escolares, do processo ensino-aprendizagem e da participação da família. Como salientam Angelucci, Kalmus, Paparelli e Patto (2004), a questão da queixa escolar é complexa e exige uma análise aprofundada e ampliada daqueles que se propõem a realizar atendimentos, pois não se pode mais isentar a escola-e, sobretudo, o professor-de responsabilidades, bem como não se pode mais continuar identificando somente a família como agente produtor das dificuldades vivenciadas pelas crianças. Guzzo (2003), por exemplo, ao discutir diversos problemas do sistema educacional brasileiro e seus agravantes sociais, como violência, marginalização e evasão, defende que tal realidade necessita ser vislumbrada sob um enfoque mais proativo e menos reativo de atendimento. Em relação à queixa e evasão escolar, Schoen-Ferreira, Silva, Farias e Silvares (2002) afirmam que abandonar a escola pode relacionar-se com a falta de motivação, falta de encorajamento parental, baixas expectativas por parte dos professores e problemas disciplinares, além dos problemas relacionados à estrutura escolar, notadamente do ensino público, principal fonte de encaminhamentos.

Nesse contexto, torna-se imperativa a atuação do psicólogo escolar/educacional no sentido de ampliar e redimensionar sua visão e seu manejo no âmbito da triagem e dos atendimentos das queixas escolares. Esta diretriz também é reforçada pelo Estatuto da Associação Brasileira de Psicologia Escolar e Educacional (ABRAPEE, 1991), no qual o psicólogo escolar é tido como um profissional que atua na escola ou em instituições vinculadas a ela, e que trabalha para melhorar o processo ensino-aprendizagem de forma global (cognitivo, emocional, social e motor) e contextual, por meio de serviços oferecidos a indivíduos, grupos, famílias e organizações. Andrada (2005) e também Rodrigues, 
Itaborahy, Pereira e Gonçalves (2008) destacam a necessidade de o psicólogo escolar redimensionar sua atuação, promovendo transformações no âmbito das práticas cristalizadoras, no sentido de programar estratégias mais contextualizadas envolvendo família e escola. Por outro lado, Silveira, Silvares e Marton (2003) defendem ser necessário e desejável que as modalidades de atuação preventiva ocorram em serviços de Psicologia, pois tendem a maximizar a adesão da clientela e minimizar os problemas comportamentais. Na mesma direção, Merg (2008) considera que o conhecimento do perfil da clientela infantil pode contribuir para melhorar o atendimento nos referidos serviços, como também fornecer subsídios relevantes para a implantação de projetos preventivos tanto no próprio contexto escolar quanto nos serviços-escola de Psicologia.

Diante do exposto, da demanda crescente de queixas escolares, do número de estagiários frequentemente alocados e, sobretudo, considerando a inexistência de estudos dessa natureza na cidade de Juiz de Fora (MG), o presente estudo propõe-se a conhecer e caracterizar as queixas escolares do Centro de Psicologia Aplicada da Universidade Federal de Juiz de Fora (CPA/UFJF), por meio da análise de prontuários. Além de nortear ações acadêmicas que incluam a supervisão de estágio na área em questão, o aperfeiçoamento do processo de triagem e a própria organização e funcionamento do CPA/UFJF, fomenta-se a expectativa de que os resultados possam contribuir para uma melhor contextualização da queixa escolar e otimização dos atendimentos a essa clientela.

\section{Método}

O trabalho envolveu uma pesquisa documental com análise quantitativa (estatística descritiva) e qualitativa. A instituição colaboradora CPA/UFJF foi escolhida de forma não probabilística, considerando-se, entre outros aspectos, a natureza pública desse serviço de atendimento psicológico à comunidade local.

\section{Instrumento}

Foi desenvolvido um software visando facilitar a 244 coleta de dados dos documentos. Os campos de entrada do software foram criados com base numa generalização dos modelos de prontuários usados pelo CPA, e com o auxílio da literatura consultada. A ferramenta contempla os seguintes dados: número do registro e do prontuário, sexo, idade, escolaridade, local de moradia, renda familiar, tipo de família, origem do encaminhamento, resolução da triagem, e tipologia das queixas apresentadas. $\mathrm{O}$ instrumento foi composto de duas partes, uma com os dados sociodemográficos, e outra com a transcrição das queixas presentes nas triagens.

\section{Procedimentos}

Após a aprovação pelo Comitê de Ética em Pesquisa da Universidade Federal de Juiz de Fora (UFJF), protocolo no 1742.086.2009, em 11/8/2009, e dispensa do Termo de Consentimento Livre e Esclarecido por tratar-se de pesquisa documental, apresentaram-se os objetivos da pesquisa para o coordenador, administradora e secretária do CPA, os quais mostraram a realidade da documentação disponível. Foram mantidas a integridade do material, a especificidade de seu arquivamento, e a confidencialidade da documentação dele derivada. Os dados sociodemográficos foram analisados a partir de uma estatística descritiva e a caracterização da queixa (foco do estudo) foi realizada a partir da análise de conteúdo temática e frequencial proposta por Bardin (2008) que pressupõe três fases: pré-análise, análise do material e o tratamento dos resultados. Assim, procedeu-se, de modo preliminar, a uma exploração de cada queixa individualmente, buscando-se expressões com o mesmo sentido e padrões (pré-análise dos conteúdos), seguindo-se com a organização do material e o procedimento de pré-categorização das mesmas (análise vertical). A partir desse procedimento, foi realizada uma aglutinação das queixas escolares recorrentes (análise horizontal), derivando-se categorias mais amplas, categorias específicas e subespecíficas que foram contabilizadas de forma bruta, apresentando-se o percentual (\%) de ocorrência de cada uma de acordo com o total de queixas identificadas. A fim de se verificar a homogeneidade das distribuições das categorias ao longo das variáveis, foi empregado o teste de Qui-Quadrado $\left(\chi^{2}\right)$, adotando-se nível de significância de $5 \%(p<0,05)$. 


\section{Resultados e Discussão}

Do ponto de vista da análise dos prontuários, vale ressaltar, inicialmente, a situação das fichas de triagem. Verificou-se durante a coleta dos dados que, entre 1996 a 2001, as fichas eram sucintas, contendo apenas dados pessoais (como nome, endereço, origem do encaminhamento) e uma síntese restritiva da queixa. Entre os anos de 2001 e 2003 os prontuários foram reformulados passando a incluir dados referentes à renda familiar, profissão, estado civil e escolaridade. No início do ano de 2009, passaram a vigorar dois tipos de fichas, uma destinada aos clientes adultos e outra para às crianças e adolescentes. Além das informações mencionadas, o histórico médico e novos dados socioeconômicos, como a renda, foram incluídos em ambas as fichas.

Constatou-se que a descrição das queixas também passou por modificações, dado que nos primeiros seis anos a transcrição contemplava poucas informações sobre o indivíduo e a queixa escolar. Nos anos seguintes, a análise dos prontuários evidenciou que houve um aprimoramento no processo de triagem e os relatos passaram a ser transcritos com maior detalhamento, o que facilitou a categorização da queixa apresentada. Durante o levantamento, verificou-se, por exemplo, que $41,80 \%$ dos documentos não possuíam informações relacionadas à renda. Nos demais prontuários, 26,48\% possuíam renda inferior a um salário-mínimo, 45,74\% entre um e três salários-mínimos e 27,78\% acima de três salários-mínimos, corroborando outros estudos nacionais, como o de Nakamura et al.
(2008), que também identificou a predominância de uma clientela de baixa renda.

Foram identificadas e analisadas 9032 fichas de triagem compreendendo os prontuários de clientes de 1996 a 2009, sendo 1590 (17,6\%) encaminhamentos específicos de queixa escolar, os quais compõem a amostra do presente estudo.

Conforme mostra a Tabela 1, a maioria dos encaminhamentos relacionados à queixa escolar refere-se ao sexo masculino (66,0\%), dado convergente com as pesquisas de Levandowski e Scortegagna (2004), Melo e Perfeito (2006), Nakamura et al. (2008), Schoen-Ferreira et al. (2002) e com os estudos de revisão realizados por Boaz e Nunes (2009) e Merg (2008). A maior frequência da população masculina foi encontrada na faixa etária de 7 a 13 anos, afinando-se com a observação de Romaro e Capitão (2003) de que tal predominância tende a ocorrer na infância e início da adolescência.

Quanto à idade, como evidencia a Tabela 1, a maior demanda compreendeu a faixa etária de 7 a 13 anos $(66,6 \%)$. Merece destaque o período de idade correspondente á primeira etapa da educação básica, a saber, entre 7 e 10 anos, que perfazem 40,0\% das queixas, convergindo com os dados encontrados por Cunha e Benetti (2009) e Melo e Perfeito (2006), que também observaram clientela escolar com faixa etária semelhante. Para Cunha e Benetti (2009), o início da escolarização poderia enfatizar os problemas emocionais ou comportamentais, uma vez que a criança se depara com uma nova realidade que lhe exige o cumprimento de determinados comportamentos.

Tabela 1.Caracterização da clientela com queixa escolar. Juiz de Fora (MG), 1996-2009.

\begin{tabular}{|c|c|c|c|c|c|c|}
\hline \multirow{3}{*}{ Faixa etária } & \multicolumn{4}{|c|}{ Sexo } & & \\
\hline & \multicolumn{2}{|c|}{ Masculino } & \multicolumn{2}{|c|}{ Feminino } & \multicolumn{2}{|c|}{ Total } \\
\hline & $n$ & $\%$ & $\mathrm{n}$ & $\%$ & $n$ & $\%$ \\
\hline 2 a 6 anos & 159 & 10,00 & 95 & 6,00 & 254 & 15,97 \\
\hline 7 a 13 anos & 727 & 45,72 & 332 & 20,88 & 1059 & 66,60 \\
\hline 14 a 19 anos & 151 & 9,50 & 96 & 6,05 & 247 & 15,53 \\
\hline$>$ de 19 anos & 13 & 0,82 & 17 & 1,07 & 30 & 1,90 \\
\hline Total & 1050 & 66,00 & 540 & 34,00 & 1590 & 100,00 \\
\hline Ano & $n$ & $\%$ & $n$ & $\%$ & $n$ & $\%$ \\
\hline 1996 a 2000 & 304 & 19,12 & 135 & 8,50 & 439 & 27,61 \\
\hline 2001 a 2005 & 511 & 32,14 & 279 & 17,54 & 790 & 49,69 \\
\hline 2006 a 2009 & 235 & 14,78 & 126 & 7,92 & 361 & 22,70 \\
\hline
\end{tabular}


A Tabela 1 evidencia ainda que a busca por atendimento psicológico referente às questões escolares foi mais frequente entre 2001 a 2005 (49,7\%), com aumento de $22,0 \%$ em relação aos quatro primeiros anos de funcionamento. Desde a implantação do CPA/UFJF a maioria dos encaminhamentos foi realizada por escolas (36,0\%), seguido por encaminhamentos referentes às instituições públicas da cidade $(34,0 \%)$ e demanda espontânea 21,7\%. Campezatto e Nunes (2007) em revisão de literatura encontraram dados semelhantes ao analisar a realidade de oito serviços-escolas de Psicologia em Porto Alegre, sendo os encaminhamentos provenientes de escolas quando se tratava de público infantil, e prevalecendo demanda espontânea para a clientela adolescente e adulta. Como mostram alguns estudos (Campezatto \& Nunes, 2007; Nakamura et al., 2008; Romaro \& Capitão, 2003; Schoen-Ferreira et al., 2002), a expressiva demanda de queixas oriundas do ensino fundamental é uma realidade dos serviços-escola de Psicologia brasileiros. Os dados aqui obtidos convergem nesta direção, visto que $74,4 \%$ dos prontuários referiam-se a estudantes do ensino fundamental, oriundos em sua maioria de regiões periféricas da cidade $(82,2 \%)$, sendo pouco frequentes, encaminhamentos provenientes de outras cidades.

No que tange de modo mais específico ás queixas escolares, foco do presente estudo, a análise de conteúdo referente aos prontuários de encaminhamentos permitiu delimitar sete categorias globais, conforme apresenta na Tabela 2. Dentre essas categorias, a coocorrência de problemas comportamentais e DA apresentou a maior frequência (30,3\%), seguida da categoria DA (26,5\%) e dos Problemas Comportamentais (24,0\%), evidenciando-se diferença significante pelo teste de Qui-Quadrado $\left(\chi^{2}=1005,239, p<0,001\right)$.

A categoria global de maior incidência, coocorrência dos problemas comportamentais e DA, derivou 5 categorias específicas. Agressividade associada à DA, foi a categoria predominante (34,0\%), seguida de ansiedade e/ou agitação associada à DA (22,0\%) e problemas de atenção associada à DA (19,7\%), sendo tal prevalência significante $\left(\chi^{2}=90,133, p<0,001\right)$.

É relevante destacar que uma análise ainda mais detalhada dessas categorias específicas reflete um quadro múltiplo e preocupante de problemas adicionais 246 que inclui: baixo desempenho, agitação e falta de motivação para os estudos, rebeldia, furtos, apatia, dentre outros. Os resultados convergem com a literatura nacional, assemelhando-se, por exemplo, aos obtidos por Nakamura et al. (2008) em estudo realizado em Rondônia, bem como com o estudo de Campezatto e Nunes (2007), realizado em Porto Alegre, onde também constatou-se predomínio de queixas coocorrentes de DA, agressividade e ansiedade. Ressalta-se que no estudo aqui apresentado, a ansiedade também aparece como queixa associada em 22,0\% dos prontuários analisados. Estes dados reforçam a afirmativa de Marturano e Loureiro (2003) e Marturano, Toller e Elias (2005) de que a coocorrência de problemas comportamentais e DA é a grande responsável pela expressiva demanda de queixas escolares nos serviços-escola de Psicologia no país.

Como mostra a Tabela 2, a segunda categoria global com maior ocorrência de queixas foi a de DA, perfazendo $26,5 \%$ do total dos encaminhamentos. A análise referente a essa categoria indicou a predominância da categoria DA, com 95,0\% dos registros $\left(\chi^{2}=348,430, p<0,001\right)$. Evidenciou-se na análise dos prontuários que os encaminhamentos referentes às DA chegam geralmente de forma inespecífica, "a criança não aprende" (43,8\%) e associados de forma indireta com lentidão, falta de motivação e problemas de socialização, que totalizaram 42,3\% das queixas adicionais. Os resultados afinam-se com os obtidos por Louzada (2003), na medida em que a DA apareceram como segunda queixa mais frequente no grupo de crianças/adolescentes.

Observa-se que tanto a coocorrência quanto as DA (isoladas) surgem associadas a outros fatores intrínsecos que tradicionalmente são imputados, de forma restritiva, ao aluno, realidade convergente com os estudos de revisão realizados sobre clínicas-escola por Levandowski (1998), Merg (2008), Silvares (1996) e por Cabral e Sawaya (2001), no setor da saúde pública, os quais também evidenciaram um contingente expressivo de encaminhamentos de queixas escolares com coocorrência de problemas comportamentais e de aprendizagem.

Como mostra a Tabela 2, a terceira tipologia de queixas - problemas comportamentais - aparece com 24,0\% da ocorrência dos registros, destacando-se dentre as categorias específicas, a agressividade (52,8\%), seguida de indisciplina e agitação (28,6\%), sendo tal prevalência significante $\left(\chi^{2}=332,451, p<0,001\right)$. 
Tabela 2. Caracterização da queixa escolar do CPA/UFJF (1996-2009).

continua...

\begin{tabular}{|c|c|c|c|c|c|c|c|c|}
\hline Categorias globais & $\mathrm{F}$ & $\%$ & Categorias específicas & $\mathrm{F}$ & $\%$ & Categorias subespecíficas & $\mathrm{F}$ & $\%$ \\
\hline \multirow{16}{*}{$\begin{array}{l}\text { Coocorrência de } \\
\text { Problemas } \\
\text { Comportamentais e } \\
\text { Dificuldade de } \\
\text { Aprendizagem }\end{array}$} & \multirow{16}{*}{481} & \multirow{16}{*}{30,25} & \multirow{5}{*}{$\begin{array}{l}\text { Relacionadas com } \\
\text { agressividade e } \\
\text { dificuldades de } \\
\text { aprendizagem }\end{array}$} & \multirow{5}{*}{164} & \multirow{5}{*}{34,10} & $\begin{array}{l}\text { Queixas de agressividade e dificuldades de } \\
\text { aprendizagem isoladas }\end{array}$ & 98 & 59,76 \\
\hline & & & & & & $\begin{array}{l}\text { Associadas com agitação e baixa motiva- } \\
\text { ção para os estudos }\end{array}$ & 48 & 29,27 \\
\hline & & & & & & Associadas as dificuldades de socialização & 14 & 8,54 \\
\hline & & & & & & Associadas a problemas familiares & 4 & 2,44 \\
\hline & & & & & & & 164 & 100,00 \\
\hline & & & \multirow{4}{*}{$\begin{array}{l}\text { Ansiedade e/ou agitação } \\
\text { com dificuldades de } \\
\text { aprendizagem }\end{array}$} & \multirow{4}{*}{106} & \multirow{4}{*}{22,04} & $\begin{array}{l}\text { Queixas de agitação e de dificuldades de } \\
\text { aprendizagem isoladas }\end{array}$ & 75 & 70,75 \\
\hline & & & & & & Queixas de baixo rendimento & 22 & 20,75 \\
\hline & & & & & & $\begin{array}{l}\text { Associadas a baixa motivação para os es- } \\
\text { tudos }\end{array}$ & 9 & 8,49 \\
\hline & & & & & & & 106 & 100,00 \\
\hline & & & $\begin{array}{l}\text { Problemas de atenção } \\
\text { associados a dificuldades } \\
\text { de aprendizagem }\end{array}$ & 95 & 19,75 & & & \\
\hline & & & \multirow[t]{4}{*}{$\begin{array}{l}\text { Indisciplina e dificuldade } \\
\text { de aprendizagem }\end{array}$} & \multirow[t]{4}{*}{81} & \multirow[t]{4}{*}{16,84} & $\begin{array}{l}\text { Queixas de indisciplina e dificuldades de } \\
\text { aprendizagem isoladas }\end{array}$ & 40 & 49,38 \\
\hline & & & & & & Associadas a rebeldia & 31 & 38,27 \\
\hline & & & & & & Associadas a furtos & 10 & 12,35 \\
\hline & & & & & & & 81 & 100,00 \\
\hline & & & $\begin{array}{l}\text { Timidez e dificuldades de } \\
\text { aprendizagem }\end{array}$ & 35 & 7,28 & $\begin{array}{l}\text { Queixas de timidez e dificuldade de apren- } \\
\text { dizagem isoladas } \\
\text { Associadas com apatia }\end{array}$ & 25 & $\begin{array}{l}71,43 \\
28,57\end{array}$ \\
\hline & & & & 481 & 100,00 & & 35 & 100,00 \\
\hline \multirow{10}{*}{$\begin{array}{l}\text { Dificuldades de } \\
\text { aprendizagem }\end{array}$} & \multirow{10}{*}{421} & \multirow{10}{*}{26,48} & \multirow{8}{*}{$\begin{array}{l}\text { Dificuldades para } \\
\text { aprender }\end{array}$} & \multirow{8}{*}{402} & \multirow{8}{*}{95,49} & $\begin{array}{l}\text { Queixas de dificuldades de aprendizagem } \\
\text { inespecíficas }\end{array}$ & 176 & 43,78 \\
\hline & & & & & & Associadas a lentidão & 59 & 14,68 \\
\hline & & & & & & $\begin{array}{l}\text { Associadas com baixa motivação para os } \\
\text { estudos }\end{array}$ & 56 & 13,93 \\
\hline & & & & & & $\begin{array}{l}\text { Associadas com dificuldade de sociali- } \\
\text { zação }\end{array}$ & 55 & 13,68 \\
\hline & & & & & & Associadas com problema familiares & 31 & 7,71 \\
\hline & & & & & & Associadas com problema neurológico & 19 & 4,73 \\
\hline & & & & & & Associadas com gagueira & 6 & 1,49 \\
\hline & & & & & & & 402 & 100,00 \\
\hline & & & Hiperatividade & 19 & 4,51 & & & \\
\hline & & & & 421 & 100,00 & & & \\
\hline
\end{tabular}

Dentre os problemas comportamentais, surgem ainda, de forma ainda mais específica e frequente, problemas relacionados à agitação, indisciplina, brigas e rebeldia. Resultados semelhantes aos que foram obtidos em outros serviços-escola universitários no país, os quais também encontraram o predomínio dessa tipo- logia de queixas escolares (Levandowski \& Scortegagna, 2004; Melo \& Perfeito, 2006; Nakamura et al., 2008).

A agressividade tem sido identificada como uma das queixas mais recorrentes e preocupantes dentre os problemas socioemocionais na medida em que, como evidenciam Marturano e Loureiro (2003), afeta tanto o 
Tabela 2. Caracterização da queixa escolar do CPA/UFJF (1996-2009).

Continuação

\begin{tabular}{|c|c|c|c|c|c|c|c|c|}
\hline Categorias globais & $\mathrm{F}$ & $\%$ & Categorias específicas & $\mathrm{F}$ & $\%$ & Categorias subespecíficas & $\mathrm{F}$ & $\%$ \\
\hline \multirow{12}{*}{$\begin{array}{l}\text { Problemas } \\
\text { Comportamentais }\end{array}$} & \multirow{12}{*}{381} & \multirow{12}{*}{23,96} & \multirow{4}{*}{$\begin{array}{l}\text { Relacionadas com } \\
\text { agressividade }\end{array}$} & \multirow{4}{*}{201} & \multirow{4}{*}{52,76} & Queixas de agressividade isoladas & 133 & 66,17 \\
\hline & & & & & & Associadas com agitação & 55 & 27,36 \\
\hline & & & & & & Associadas com disritmia & 13 & 6,47 \\
\hline & & & & & & & 201 & 100,00 \\
\hline & & & \multirow{4}{*}{$\begin{array}{l}\text { Relacionadas com } \\
\text { indisciplina e agitação }\end{array}$} & \multirow{4}{*}{109} & \multirow{4}{*}{28,61} & Queixas de indisciplina ou agitação isoladas & 90 & 82,57 \\
\hline & & & & & & Rebeldia & 10 & 9,17 \\
\hline & & & & & & Brigas & 9 & 8,26 \\
\hline & & & & & & & 109 & 100,00 \\
\hline & & & $\begin{array}{l}\text { Baixa } \\
\text { concentração/dispersão }\end{array}$ & 39 & 10,24 & & & \\
\hline & & & Ansiedade & 21 & 5,51 & & & \\
\hline & & & Apatia & 11 & 2,89 & & & \\
\hline & & & & 381 & 100,00 & & & \\
\hline \multirow{17}{*}{$\begin{array}{l}\text { Problemas/ } \\
\text { Dificuldades Gerais }\end{array}$} & \multirow{17}{*}{166} & \multirow{17}{*}{10,44} & \multirow{4}{*}{$\begin{array}{l}\text { Relacionadas com } \\
\text { dificuldades de } \\
\text { adaptação }\end{array}$} & \multirow[t]{4}{*}{55} & \multirow[t]{4}{*}{33,13} & $\begin{array}{l}\text { Queixas relacionadas ao constrangimento } \\
\text { e à aversão escolar }\end{array}$ & 38 & 69,09 \\
\hline & & & & & & Associadas com gagueira & 12 & 21,82 \\
\hline & & & & & & $\begin{array}{l}\text { Queixas gerais inespecíficas de dificuldades } \\
\text { de adaptação }\end{array}$ & 5 & 9,09 \\
\hline & & & & & & & 55 & 100,00 \\
\hline & & & \multirow{4}{*}{$\begin{array}{l}\text { Relacionadas com outros } \\
\text { problemas de saúde } \\
\text { física/mental }\end{array}$} & \multirow[t]{4}{*}{52} & \multirow[t]{4}{*}{31,33} & Problemas gerais de saúde & 29 & 55,77 \\
\hline & & & & & & Problemas neurológicos/orgânicos & 14 & 26,92 \\
\hline & & & & & & Deficiência mental/deficit cognitivo & 9 & 17,31 \\
\hline & & & & & & & 52 & 100,00 \\
\hline & & & \multirow{4}{*}{$\begin{array}{l}\text { Relacionadas com } \\
\text { problemas sensoriais }\end{array}$} & \multirow{4}{*}{28} & \multirow{4}{*}{16,87} & Dificuldade na fala & 20 & 71,43 \\
\hline & & & & & & Deficiência auditiva & 6 & 21,43 \\
\hline & & & & & & Deficiência visual & 2 & 7,14 \\
\hline & & & & & & & 28 & 100,00 \\
\hline & & & $\begin{array}{l}\text { Problemas escolares mais } \\
\text { gerais }\end{array}$ & 14 & 8,43 & & & \\
\hline & & & $\begin{array}{l}\text { Desorganização e hábitos } \\
\text { deficitários de estudo }\end{array}$ & 11 & 6,63 & & & \\
\hline & & & $\begin{array}{l}\text { Hipótese de aluno } \\
\text { superdotado ou } \\
\text { talentoso }\end{array}$ & 4 & 2,41 & & & \\
\hline & & & $\begin{array}{l}\text { Avaliação visando } \\
\text { antecipação na } \\
\text { escolaridade }\end{array}$ & 2 & 1,20 & & & \\
\hline & & & & 166 & 100,00 & & & \\
\hline
\end{tabular}

convívio social do aluno quanto o seu desempenho escolar, embora em muitos casos tais queixas não apareçam relacionadas no motivo do encaminhamento (Barros \& Silva, 2006; Marturano et al., 2005; Santos \&

248 Graminha, 2006). Ressalta-se que estudos internacionais e nacionais identificam a agressividade como fator de risco importante na trajetória de desenvolvimento individual da criança (Lisboa \& Koller, 2001; Maia \&Willians, 2005; Severson, Walker, Hope-Doolittle, Kratochwill \& Gresham, 2007), reforçando a preocupação inserida na 
Tabela 2. Caracterização da queixa escolar do CPA/UFJF (1996-2009).

Conclusão

\begin{tabular}{|c|c|c|c|c|c|c|c|c|}
\hline Categorias globais & F & $\%$ & Categorias específicas & $\mathrm{F}$ & $\%$ & Categorias subespecíficas & $\mathrm{F}$ & $\%$ \\
\hline \multirow{10}{*}{$\begin{array}{l}\text { Problemas de Ordem } \\
\text { Afetivo-Emocional }\end{array}$} & \multirow{10}{*}{65} & \multirow{10}{*}{4,09} & \multirow{4}{*}{$\begin{array}{l}\text { Relacionadas com } \\
\text { instabilidade emocional }\end{array}$} & \multirow{4}{*}{29} & \multirow{4}{*}{44,62} & Choro constante & 22 & 75,86 \\
\hline & & & & & & Tristeza e baixa autoestima & 4 & 13,79 \\
\hline & & & & & & Queixas gerais de instabilidade emocional & 3 & 10,34 \\
\hline & & & & & & & 29 & 100,00 \\
\hline & & & \multirow{4}{*}{$\begin{array}{l}\text { Co-ocorrência de } \\
\text { problemas emocionais } \\
\text { com dificuldade de } \\
\text { aprendizagem }\end{array}$} & \multirow{4}{*}{18} & \multirow{4}{*}{27,69} & $\begin{array}{l}\text { Outros problemas emocionais associados } \\
\text { com dificuldades escolares }\end{array}$ & 7 & 38,89 \\
\hline & & & & & & Carência e baixo rendimento & 6 & 33,33 \\
\hline & & & & & & Insegurança e lentidão & 5 & 27,78 \\
\hline & & & & & & & 18 & 100,00 \\
\hline & & & \multirow[t]{2}{*}{$\begin{array}{l}\text { Excessiva dependência } \\
\text { do adulto }\end{array}$} & 18 & 27,69 & $\begin{array}{l}\text { Associada com medo } \\
\text { Associada com queda no rendimento } \\
\text { Queixas de excessiva dependência isoladas }\end{array}$ & $\begin{array}{l}9 \\
7 \\
2\end{array}$ & $\begin{array}{l}50,00 \\
38,89 \\
11,11\end{array}$ \\
\hline & & & & 65 & 100,00 & & 18 & 100,00 \\
\hline \multirow{4}{*}{$\begin{array}{l}\text { Problemas no } \\
\text { Relacionamento } \\
\text { Interpessoal }\end{array}$} & \multirow{4}{*}{61} & \multirow{4}{*}{3,84} & $\begin{array}{l}\text { Queixas de dificuldades } \\
\text { de socialização }\end{array}$ & 46 & 75,41 & & & \\
\hline & & & $\begin{array}{l}\text { Associadas com } \\
\text { agressividade e agitação }\end{array}$ & 10 & 16,39 & & & \\
\hline & & & Associadas com timidez & 5 & 8,20 & & & \\
\hline & & & & 61 & 100,00 & & & \\
\hline \multirow{3}{*}{$\begin{array}{l}\text { Dificuldades } \\
\text { Psicomotoras }\end{array}$} & \multirow{3}{*}{15} & \multirow{3}{*}{0,94} & $\begin{array}{l}\text { Associadas com } \\
\text { dificuldade de } \\
\text { aprendizagem }\end{array}$ & 10 & 66,67 & & & \\
\hline & & & $\begin{array}{l}\text { Associadas com } \\
\text { agressividade e baixa } \\
\text { motivação }\end{array}$ & 3 & 20,00 & & & \\
\hline & & & $\begin{array}{l}\text { Queixas de dificuldade } \\
\text { psicomotoras isoladas }\end{array}$ & 2 & 13,33 & & & \\
\hline Total & 1590 & 100,00 & & 15 & 100,00 & & & \\
\hline
\end{tabular}

revisão de Merg (2008) e nos estudos realizados por Silvares (1993) e Silveira et al. (2003) quanto á implementação de estratégias mais proativas no âmbito dos serviços-escola universitários. Considera-se que tais estratégias, que necessitam ser implementadas também pelas escolas, como salientam Cunha e Rodrigues (2010), poderiam minimizar fatores de risco e fomentar fatores de proteção ao desenvolvimento infantil, como já discutiram Graminha, Martins e Miura (1996).

De acordo com a Tabela 2, a quarta categoria global de queixas delimitada pela análise dos prontuários, problemas/dificuldades gerais, refletem em sua maioria questões relacionadas com dificuldades mais gerais de adaptação ao ambiente escolar $(33,0 \%)$ e problemas de saúde física/mental $(31,5 \%)\left(\chi^{2}=122,855\right.$, $p<0,001)$. Dificuldades mais gerais como problemas de adaptação, constrangimento e aversão escolar também foram encontradas por Schoen-Ferreira et al. (2002). Os problemas de saúde física/mental (neurológicos, deficit cognitivo, por exemplo) também foram encontrados, embora com menor frequência, por Graminha et al. (1996) e por Levandowski e Scortegagna (2004).

Quanto à categoria global problemas de ordem afetivo-emocional, delimitou-se 3 categorias específicas relacionadas predominantemente com: instabilidade emocional $(44,6 \%)$, coocorrência de problemas emocionais e DA $(27,7 \%)$ e excessiva dependência do adulto 
$(27,7 \%)\left(\chi^{2}=3,723, p\right.$-valor=0,155). Expressiva frequência de problemas emocionais também foi encontrada nos estudos de Levandowski e Scortegagna (2004) e de Melo e Perfeito (2006). Em análise mais minuciosa das fichas de triagem, foram encontradas variáveis referentes à autopercepção, tais como queixa de baixa autoestima, carência, insegurança e dependência, similar ao estudo de Marturano e Loureiro (2003), o qual evidenciou alta incidência de coocorrência de fatores socioemocionais eDA.

Conforme apresenta a Tabela 2, a sexta categoria mais geral - problemas no relacionamento interpessoal - derivou três categorias específicas, com destaque prevalente para as queixas de dificuldades de socialização (75,4\%), seguida de queixas menos frequentes associadas com agressividade e agitação $(16,4 \%)$ $\left(\chi^{2}=49,213, p<0,001\right)$. Problemas externalizantes foram também recorrentes nas pesquisas realizadas por Marturano e Loreiro (2003) e Marturano et al. (2005). Destaca-se que dificuldades no âmbito do relacionamento interpessoal também surgem em vários estudos nacionais, dentre eles Melo e Perfeito (2006), Romaro e Capitão (2003) e Schoen-Ferreira et al. (2002), na região Sudeste; Campezatto e Nunes (2007) e Levandowski e Scortegagna (2004), na região Sul; além de Nakamura et al. (2008), na região Norte.

Dificuldades psicomotoras foi a categoria global menos identificada como queixa nos prontuários, perfazendo cerca de $1 \%$ dos encaminhamentos. Por fim, $66,8 \%$ dos registros surgem associados às DA, com diferença significante sobre as demais categorias $\left(\chi^{2}=7,60, p=0,022\right)$, afinando-se com os estudos de Cabral e Sawaya (2001) e Levandowski e Scortegagna (2004).

\section{Considerações Finais}

A presente pesquisa evidenciou uma realidade convergente com a literatura nacional, que destaca a recorrência do perfil de clientela com queixa escolar encaminhado aos serviços-escola de Psicologia brasileiros.

Torna-se oportuno salientar, diante do panorama de resultados, a importância da presença do psicólogo ou de consultorias eventuais, que auxiliem os agentes educativos na implementação e promoção de estraté-

250 gias psicoeducacionais mais reflexivas e contextuali- zadas no âmbito escolar, na medida em que podem contribuir para evitar encaminhamentos e/ou agravamento das queixas escolares. É possível considerar a relevância da implementação de estratégias preventivas de cunho psicossocial, haja vista o expressivo contingente de queixas relacionadas á agressividade e outros problemas socioemocionais aqui identificados. Nesse sentido, a postura dos próprios psicólogos atuantes nas escolas frente aos problemas escolares ainda constitui um desafio. Conforme foi discutido no presente trabalho, ainda há uma ênfase em práticas limitadoras e reativas por parte desses profissionais, inclusive junto aos professores, que representam muitas vezes a fonte prioritária dos encaminhamentos. Sobre a queixa escolar compartilha-se da visão conclusiva de Levandowski e Scortegagna (2004), que defendem a abertura de um espaço de reflexão por parte dos psicólogos no contexto escolar, com uma participação mais efetiva junto ao professor pois este necessita ser ouvido e esclarecido a fim de que possa visualizar sua competência para ajudar e agir em benefício do seu aluno.

Por outro lado, do ponto de vista da organização e funcionamento do CPA/UFJF, embora tenha sido observado um aperfeiçoamento dos prontuários ao longo do período investigado, constata-se que o processo de triagem, necessita ser redimensionado e qualificado na direção de uma maior contextualização da queixa escolar apresentada - sobretudo quando se trata de encaminhamento oriundo da escola. Muitas vezes, faltam informações relevantes e mais específicas quanto ao motivo do encaminhamento e, por exemplo, uma avaliação mais detalhada quanto á percepção dos pais e da criança/adolescente quanto ao problema apresentado.

Embora considerando as limitações de pesquisa documental desta natureza (análise de prontuários oriundos de triagens realizadas por alunos ainda em formação), almeja-se que os dados obtidos possibilitem um aprimoramento das entrevistas iniciais, no sentido de ampliar o escopo da queixa apresentada e favorecer as discussões acadêmicas tecidas nas reuniões de triagem realizadas no serviço-escola.

Espera-se, de modo complementar, que o panorama aqui delineado forneça subsídios relevantes para a literatura nacional na área e, de modo mais específico, para fomentar projetos de pesquisa e extensão voltados 
para o melhor atendimento da clientela com queixa escolar do CPA/UFJF, bem como novas diretrizes para os projetos universitários participativos que envolvam a atuação em psicologia escolar/educacional em escolas da rede de ensino de Juiz de Fora (MG).

\section{Referências}

Andrada, E. G. C. (2005). Novos paradigmas na prática do psicólogo escolar. Psicologia Reflexão e Crítica, 18 (2), 196-199.

Ancona-Lopez, M. (1983). Características da clientela de clínicas-escola de Psicologia de São Paulo. Arquivos Brasileiros de Psicologia, 1 (35), 78-92.

Angelucci, C. B., Kalmus, J., Paparelli, R., \& Patto, M. H. S. (2004). O estado da arte da pesquisa sobre o fracasso escolar (1991-2002): um estudo introdutório. Educação e Pesquisa, 30 (1), 51-72.

Associação Brasileira de Psicologia Escolar e Educacional. (1991). Estatuto da Associação Brasileira de Psicologia Escolar e Educacional. Campinas: ABRAPEE .

Barbosa, J. I., \& Silvares, E. F. (1994). Uma caracterização preliminar das clínicas-escola de Fortaleza. Estudos de Psicologia (Campinas), 11 (3), 50-56.

Bardin, L. (2008). Análise de conteúdo. Lisboa: Edições 70.

Barros, P., \& Silva, F. B. N. (2006). Origem e manutenção do comportamento agressivo na infância e adolescência. Revista Brasileira de Terapia Cognitiva, 2 (1), 55-56.

Boarini, M. L., \& Borges, R. F. (1998). Demanda infantil por serviços de saúde mental: sinal de crise. Estudos de Psicologia, 3 (2), 83-108.

Boaz, C., \& Nunes, M. L. P. (2009). Caracterização das queixas de meninos e meninas em clínicas-escola nos últimos 30 anos. Anais da IV Mostra de Pesquisa da Pós-Graduação da PUC. Porto Alegre, RS.

Braga, S. G., \& Morais, M. L. S. (2007). Queixa escolar: atuação do psicólogo e interfaces com a educação. Psicologia USP, $18(4), 35-51$.

Cabral, E., \& Sawaya, S. M. (2001). Concepções e atuação profissional diante das queixas escolares: os psicólogos nos serviços públicos de saúde. Estudos de Psicologia, 6 (2), 143-155.

Collares, C. A. L., \& Moysés, M. A. A. (1996). Preconceitos no cotidiano escolar:ensino emedicalização. São Paulo: Cortez.

Campezatto, P., \& Nunes, M. L. T. (2007). Caracterização da clientela das clínicas-escolas da região metropolitana de Porto Alegre. Psicologia Reflexão e Crítica, 20 (3), 339-396.

Cunha, T. R. S., \& Benetti, S. P. C. (2009). Caracterização da clientela infantil numa clínica-escola de psicologia. Boletim de Psicologia, 59 (130), 117-127.

Cunha, N., \& Rodrigues, M. C. (2010). O desenvolvimento de competências psicossociais como fator de proteção ao desenvolvimento infantil. Estudos Interdisciplinares em Psicologia, 1 (2), 235-248.
Graminha, S. S. V. (1994). Problemas emocionais/comportamentais em uma amostra de escolares: incidência em função do sexo e idade. Psico, 25 (1), 49-74.

Graminha, S. S. V., \& Martins, M. A. Q. (1994). Procura por atendimento psicológico para crianças: características da problemática relatada pelos pais. Psico, 25 (2), 53-79.

Graminha, S. S. V., Martins, M. A. Q., \& Miura, R. T. (1996). Fatores potenciais de risco em crianças com problemas emocionais/comportamentais. Psico, 27 (2), 153-175.

Guzzo, R. S. L. (2003). Saúde psicológica, sucesso e eficácia da escola: desafios do novo milênio para a psicologia escolar. In Z. A. P. Del Prette (Org.), Psicologia escolar e educacional, saúde equalidade devida: explorando fronteiras (pp.25-42). Campinas: Alínea.

Levandowski, D. C. (1998). Caracterização da população atendida por clínica-escola: breve revisão da literatura nacional. Torre de Babel, 5 (1/2), 87-110.

Levandowski, D. C., \& Scortegagna, P. (2004). Análise dos encaminhamentos de crianças com queixa escolar da rede municipal de ensino de Caxias do Sul. Interação, 9 (18), 127-152.

Lisboa, C. S. M., \& Koller, S. H. (2001). Construção e validação de conteúdo da escala de percepção de professores dos comportamentos agressivos de crianças na escola. Psicologia em Estudo, 6 (1), 59-69.

Louzada, R. C. R. (2003). Caracterização da clientela atendida no Núcleo de Psicologia Aplicada da Universidade Federal do Espírito Santo. Estudos de Psicologia, 8 (3), 451-457.

Maia, J. M. D., \& Williams, L. C. A. (2005). Fatores de risco e fatores de proteção ao desenvolvimento infantil: uma revisão da área. Temas em Psicologia, 13 (2), 91-103.

Marturano, E. M., Linhares, M. B. M., Loureiro, S. R., \& Machado, V. S. L. (1997). Crianças referidas para baixo atendimento psicológico em virtude de baixo rendimento escolar: comparação com alunos não referidos. Revista Interamericana de Psicologia, 31 (2), 223-241.

Marturano, E. M., \& Loureiro, S. R. O. (2003). O. desenvolvimento socioemocional e as queixas escolares. In A. Del Prette \& Z. A. P. Del Prette (Orgs.), Habilidades sociais, desenvolvimento e aprendizagem. Campinas: Alínea.

Marturano, E. M., Toller, G. P., \& Elias, L. C. S. (2005). Gênero, adversidade e problemas socioemocionais associados à queixa escolar. Estudos de Psicologia (Campinas), 22 (4), 371-380. doi: 10.1590/S0103-166X2005000400005.

Melo, S. A., \& Perfeito, H. C. C. S. (2006). Características da população infantil atendida em triagem no período de 2000 a 2002 numa clínica-escola. Estudos de Psicologia (Campinas), 23 (3), 239-249. doi: 10.1590/S0103-166X2 006000300003

Merg, M. M. G. (2008). Características da clientela infantil em clínica-escola. Dissertação de mestrado não-publicada, Pontifícia Universidade Católica do Rio Grande do Sul, Porto Alegre.

Moysés, M. A. A., \& Collares, C. A. (1992). A história não contada dos distúrbios de aprendizagem. Cadernos CEDES, 28, $31-48$. 
Muñiz, A. M. R. (2001). Pediatria e psicopedagogia: parceria na avaliação do desenvolvimento da criança. Revista Psicopedagogia, 19 (58), 30-32.

Nakamura, M. S., Lima, V. A. A., Tada, I. N. C., \& Junqueira, M. H. R. (2008). Desvendando a queixa escolar: um estudo no serviço de psicologia da Universidade Federal de Rondônia. Psicologia Escolare Educacional, 12 (2), 423-429.

Rodrigues, M. C., Itaborahy, C. Z., Pereira, M. D., \& Gonçalves, T. M. (2008). Prevenção e promoção de saúde na escola: concepções e práticas de psicólogos escolares. Gerais: Revista Interinstitucional de Psicologia, 1 (11), 67-78.

Romaro, R. A., \& Capitão, C. G. (2003). Caracterização da clientela da clínica-escola de psicologia da Universidade São Francisco. Psicologia: Teoria e Prática, 5 (1), 111-121.

Santos, M. A. (1990). Caracterização da clientela de uma clínica psicológica da prefeitura de São Paulo. Arquivos Brasileiros de Psicologia, 42 (2), 79-94.

Santos, P. L., \& Graminha, S. S. V. (2006). Problemas emocionais e comportamentais associados ao baixo rendimento acadêmico. Estudos de Psicologia, 11 (1), 101-109.

Savalhia, J. A. D., \& Nunes, M. L. T. (2007). Motivos de consulta em crianças de clínicas-escola e serviços de psicologia. Revista Ciências Humanas Frederico Westphalen, 8 (11), 157-171.

Schoen-Ferreira, T. H., Silva, D. A.; Farias, M. A., \& Silvares, E. F. M. (2002). Perfil e principais queixas dos clientes encaminhados ao Centro de Atendimento e Apoio Psicológico ao Adolescente (CAAA). Psicologia em Estudo, 7 (2), 73-82.
Severson. H. H., Walker, H. M., Hope-Doolittle, J., Kratochwill, T. R., \& Gresham, F. M. (2007). Proactive, early screening to detect behaviorally at-risk students: issues, approaches, emerging innovations, and professional practices. Journal of School Psychology, 45 (2), 193-223.

Silvares, E. F. M. (1993). O papel preventivo das clínicasescola de psicologia em seu atendimento a crianças. Temas em Psicologia, 2 (1), 87-97.

Silvares, E. F. M. (1996). É satisfatório o atendimento psicológico nas clínicas-escola brasileiras? In R. M. L. L. Carvalho (Org.), Repensando a formação do psicólogo: da informação à descoberta (Coletâneas da ANPEPP). Campinas: Alínea, 1996.

Silveira, J. M., Silvares, E. F. M., \& Marton, S. A. (2003). Programas preventivos de comportamentos anti-sociais: dificuldades na pesquisa e na implementação. Estudos de Psicologia (Campinas), 20 (3), 59-67. doi: 10.1590/S0103-16 6X20030000300005.

Souza, S. M. Z. L. (1997). Avaliação escolar na formação de psicólogos: desafios e perspectivas. In J. G. Aquino (Org.), Erro efracasso na escola: alternativas teóricas e práticas. São Paulo: Summus.

Souza, B. P. (2007). Orientação à queixa escolar. São Paulo: Casa do Psicólogo.

Recebido em: 20/10/2010

Versão final reapresentada em: 9/9/2011

Aprovado em: 27/9/2011 